\title{
CLASSICAL SIMPLE LIE 2-ALGEBRAS OF ODD TORAL RANK AND A CONTRAGREDIENT LIE 2-ALGEBRA OF TORAL RANK 4
}

\author{
CARLOS R. PAYARES GUEVARA AND FABIÁN A. ARIAS AMAYA
}

\begin{abstract}
After the classification of simple Lie algebras over a field of characteristic $p>3$, the main problem not yet solved in the theory of finite dimensional Lie algebras is the classification of simple Lie algebras over a field of characteristic 2. The first result for this classification problem ensures that all finite dimensional Lie algebras of absolute toral rank 1 over an algebraically closed field of characteristic 2 are soluble. Describing simple Lie algebras (respectively, Lie 2-algebras) of finite dimension of absolute toral rank (respectively, toral rank) 3 over an algebraically closed field of characteristic 2 is still an open problem. In this paper we show that there are no classical type simple Lie 2-algebras with toral rank odd and furthermore that the simple contragredient Lie 2-algebra $G\left(F_{4, a}\right)$ of dimension 34 has toral rank 4 . Additionally, we give the Cartan decomposition of $G\left(F_{4, a}\right)$.
\end{abstract}

\section{INTRODUCTION}

The classification of the simple Lie algebras over an algebraically closed field of characteristic $p$ with $p \in\{2,3\}$ is still an open problem. In characteristic 2, S. Skryabin showed in [10] that all simple Lie algebras on an algebraically closed field of characteristic 2 have absolute toral rank greater than or equal to 2 (see also [3]). Later, A. Premet and A. Grishkov classified Lie algebras of absolute toral rank 2. They announced in [2] (work in progress) the following result: All finite dimensional simple Lie algebras over an algebraically closed field of characteristic 2 of absolute toral rank 2 are classical of dimension $3,8,14$ or 26 . In particular, every finite dimensional simple Lie 2-algebra over a field of characteristic 2 of (relative) toral rank 2 is isomorphic to $A_{2}, G_{2}$ or $D_{4}$. When the absolute rank is greater than or equal to 3 the problem of classification is still open. The main obstacle in this problem is the lack of examples.

In this paper we calculate the toral rank of the classical simple Lie 2-algebras of type $X_{l} \in\left\{A_{l}, B_{l}, C_{l}, D_{l}, \mathfrak{g}_{2}, \mathfrak{f}_{4}, \mathfrak{e}_{6}, \mathfrak{e}_{7}, \mathfrak{e}_{8}\right\}$, i.e., quotients of Chevalley algebras over

2020 Mathematics Subject Classification. 17B50, 17B20, $17 \mathrm{~B} 05$.

Key words and phrases. Simple Lie 2-algebra; toral rank; classical type Lie algebra; contragredient Lie algebra. 
a field of characteristic 2, modulo the center. As a consequence, we obtain our first main result:

Theorem 1. There are no classical type simple Lie 2-algebras of odd toral rank. In particular, there are no classical type simple Lie 2-algebras of toral rank 3.

V. Kac in [7] showed that for $p>3$ every simple finite dimensional contragredient Lie algebra is isomorphic to one of the simple Lie algebras of the classical type. If $p=2$, this is no longer true and the classification of simple finite dimensional contragredient Lie algebras is still considered an open problem. In the last section we prove that the simple contragredient Lie 2-algebra of dimension 34 constructed by V. Kac and V. Veĭsfeller in 13 has toral rank 4 and we calculate the dimension of the root spaces of this contragredient Lie algebra. More specifically, we have:

Theorem 2. The simple contragredient Lie 2-algebra of dimension 34 with Cartan matrix

$$
F_{4, a}:=\left(\begin{array}{cccc}
0 & 1 & 0 & 0 \\
a & 0 & 1 & 0 \\
0 & 1 & 0 & 1 \\
0 & 0 & 1 & 0
\end{array}\right),
$$

which is denoted by $G\left(F_{4, a}\right)$, has toral rank 4 . Furthermore, the Cartan decomposition of $G\left(F_{4, a}\right)$ with respect to the 4 -dimensional torus $T(\mathfrak{h})$ is

$$
G\left(F_{4, a}\right)=T(\mathfrak{h}) \oplus \bigoplus_{\xi \in G} \mathfrak{g}_{\xi},
$$

where $G:=\langle\alpha, \beta, \gamma, \lambda\rangle$ is an elementary abelian group of order 16 and $\operatorname{dim}_{K}\left(\mathfrak{g}_{\xi}\right)=$ 2 , for all $\xi \in G$.

(The two theorems above are presented later as Theorems 5.7 and 6.2 respectively.)

The only classical type simple Lie 2 -algebras of toral rank 4 over an algebraically closed field of characteristic 2 are the following: $\mathfrak{s l}_{5}(K), \mathfrak{p s l}_{6}(K), \mathfrak{s p}_{10}(K)^{(2)}$, and $\mathfrak{s p}_{12}(K)^{(2)} / \mathfrak{z}\left(\mathfrak{g l}_{12}(K)\right.$ ) (see Corollary 5.6). Theorem 2 gives us an example of a non-classical simple Lie 2-algebra, which should be taken into account in future investigations related to the problem of classifying the simple Lie 2-algebras of toral rank 4.

In section 1 we present some basic definitions and well-known results that will be used throughout the work. In Sections 2 and 3 we show that the linear special Lie algebra $\mathfrak{s l}_{n+1}(K)$ and the symplectic Lie algebra $\mathfrak{s p}_{2 m}(K)$ over an algebraically closed field of characteristic 2 are Lie 2-algebras, and we study the simplicity of these algebras (Theorem 2.2 and 3.4). In section 4 we show that the orthogonal Lie algebra $\mathfrak{o}_{n}(K)^{(1)}$ is not a Lie 2-algebra. In section 5 we list all classical type simple Lie 2-algebras, we calculate their toral rank, and we conclude that there are no classical type simple Lie 2-algebras with odd toral rank. Finally, in the last section we show that the simple Lie 2-algebra of dimension 34 constructed by V. Kac and V. Veľsfeller in [13] has toral rank 4, and we also give the Cartan decomposition of this algebra. 


\section{Preliminaries}

Throughout this paper all algebras are defined over a fixed algebraically closed field $K$ of characteristic 2 containing the prime field $\mathbb{K}_{2}$ and $\mathfrak{g}$ is any Lie algebra of finite dimension on $K$. We start with some basic definitions and known facts.

1.1. Simple Lie 2-algebras. The concept of Lie 2-algebra, introduced by N. Jacobson (see [5]), is as follows:

Definition 1.1. A Lie 2-algebra is a pair $(\mathfrak{g},[2])$ where $\mathfrak{g}$ is a Lie algebra over $K$ and $[2]: \mathfrak{g} \rightarrow \mathfrak{g}, a \mapsto a^{[2]}$ is a map (called 2-map) such that:

(1) $(a+b)^{[2]}=a^{[2]}+b^{[2]}+[a, b], \quad$ for all $a, b \in \mathfrak{g}$;

(2) $(\lambda a)^{[2]}=\lambda^{2} a^{[2]}$, for all $\lambda \in K, a \in \mathfrak{g}$;

(3) $\operatorname{ad}\left(b^{[2]}\right)=(\operatorname{ad}(b))^{2}, \quad$ for all $b \in \mathfrak{g}$.

If the center $\mathfrak{z}(\mathfrak{g})$ of $\mathfrak{g}$ is zero and a 2 -map [2] $\mathfrak{g} \rightarrow \mathfrak{g}$ exists, it is unique. A Lie 2-algebra $(\mathfrak{g},[2])$ is called a simple Lie 2-algebra if $\mathfrak{g}$ is a simple Lie algebra on $K$.

Example 1.2. Let $A$ be an associative algebra and let Lie $(A)$ be the Lie algebra with bracket $[x, y]=x y-y x$ for $x, y \in \operatorname{Lie}(A)$ associated with $A$. Then, $\operatorname{Lie}(A)$ is a Lie 2-algebra with $a^{[2]}:=a^{2}$. In particular, $\operatorname{Lie}(\operatorname{End}(V)):=\mathfrak{g l}(V)$ is a Lie 2-algebra, where $\operatorname{End}(V)$ is the associative algebra of $K$-endomorphisms on $V$.

Example 1.3. Let $b: V \times V \rightarrow K$ be a bilinear form and consider the subset $\mathfrak{g}(V, b)$ of $\mathfrak{g l}(V)$ defined by

$$
\mathfrak{g}(V, b):=\{f \in \mathfrak{g l}(V): b(f(u), v)+b(u, f(v))=0 \text { for all } u, v \in V\} .
$$

It is easy to prove that $(\mathfrak{g}(V, b),[2])$ is a Lie subalgebra of $(\mathfrak{g l}(V),[2])$. Moreover, for $f \in \mathfrak{g l}(V)$ and $v, w \in V$, we have

$$
b\left(f^{[2]}(v), w\right)=b(f(f(v)), w)=b(f(v), f(w))=b(v, f(f(w)))=b\left(v, f^{[2]}(w)\right) .
$$

So, $(\mathfrak{g}(V, b),[2])$ is a Lie 2 -subalgebra of $(\mathfrak{g}(V),[2])$.

For our purposes, it will be useful to have the matricial version of $\mathfrak{g}(V, b)$. Given $A \in \mathfrak{g l}_{n}(K)$, we consider

$$
\mathfrak{g}(A)=\left\{X \in \mathfrak{g l}_{n}(K): X^{T} A=A X\right\} .
$$

Let $\Theta=\left\{v_{1}, v_{2}, \ldots, v_{n}\right\}$ be a basis of $V$ and assume that $A \in \mathfrak{g l}_{n}(K)$ is the Gram matrix of $b$ with respect to the basis $\Theta$, that is,

$$
a_{i j}=b\left(v_{i}, v_{j}\right), \quad 1 \leq i, j \leq n .
$$

Then, the Lie isomorphism $T_{\Theta}: \mathfrak{g l}(V) \rightarrow \mathfrak{g l}_{n}(K)$ (which sends every $f \in \mathfrak{g l}(V)$ to its matrix $T_{\Theta}(f)$ relative to $\left.\Theta\right)$ maps $\mathfrak{g}(V, b)$ onto $\mathfrak{g}(A)$.

Two matrices $A, B \in \mathfrak{g l}_{n}(K)$ are said to be congruent if there is $S \in \mathrm{GL}_{n}(K)$ such that

$$
S^{T} A S=B
$$

In this case, the map $\mathfrak{g}(A) \rightarrow \mathfrak{g}(B)$ given by $X \mapsto S^{-1} X S$ is a Lie 2-isomorphism. 


\subsection{Maximal tori and toral rank.}

Definition 1.4. Let $(\mathfrak{g},[2])$ be a Lie 2-algebra. An element $t \in \mathfrak{g}$ is said to be a toral element if $t^{[2]}=t$. A 2-subalgebra $\mathfrak{t}$ of $(\mathfrak{g},[2])$ is called toral (or a torus of $\mathfrak{g}$ ) if the 2-mapping is invertible on $t$.

Any toral subalgebra of $\mathfrak{g}$ is abelian and admits a basis consisting of toral elements (see e.g. [5, Theorem 13, p. 192]). A torus $\mathfrak{t}$ of $\mathfrak{g}$ is called maximal if the inclusion $\mathfrak{t} \subseteq \mathfrak{t}^{\prime}$ with $\mathfrak{t}^{\prime}$ toral implies $\mathfrak{t}^{\prime}=\mathfrak{t}$.

Let $(\mathfrak{g},[2])$ be a simple Lie 2-algebra over an algebraically closed field $K$ and let $\mathfrak{h}$ be a Cartan subalgebra. The set of toroidal elements in $\mathfrak{h}$ generates a torus. We denote this torus by the symbol $T(\mathfrak{h})$. The torus $T(\mathfrak{h})$ is maximal in $(\mathfrak{g},[2])$ (see [1. Lemma 4]).

Definition 1.5 (See [12]). The toral rank of a Lie 2-algebra $(\mathfrak{g},[2])$ is

$$
M T(\mathfrak{g}):=\max \left\{\operatorname{dim}_{K}(\mathfrak{t}): \mathfrak{t} \text { is a torus in } \mathfrak{g}\right\} .
$$

\section{The SPeCial Linear Lie 2-ALGebra $\left(\mathfrak{s l}_{n+1}(K),[2]\right)$}

In this section we consider the Lie algebra consisting of matrices of trace zero over $K$, and we study some properties concerning the simplicity of this algebra.

It is a known fact that the commutator of the Lie general algebra $\mathfrak{g l}_{n+1}(K)$ is a Lie subalgebra of $\mathfrak{g l}_{n+1}(K)$. This algebra is called the special Lie algebra, and it is denoted by $\mathfrak{s l}_{n+1}(K)$. That is,

$$
\mathfrak{s l}_{n+1}(K):=\left[\mathfrak{g l}_{n+1}(K), \mathfrak{g l}_{n+1}(K)\right] .
$$

It is easy to prove that

$$
\mathfrak{s l}_{n+1}(K)=\left\{A \in \mathfrak{g l}_{n+1}(K): \operatorname{tr}(A)=0\right\} .
$$

A basis for $\mathfrak{s l}_{n+1}(K)$ is the following:

$$
h_{k}:=e_{k k}+e_{k+1, k+1}, \quad k=1, \ldots, n ; \quad e_{i j}, \quad i \neq j=1,2, \ldots, n+1 .
$$

Let us consider the 2-map [2]: $\mathfrak{s l}_{n+1}(K) \rightarrow \mathfrak{s l}_{n+1}(K)$ given by $A^{[2]}:=A^{2}$.

Remark 2.1. The Lie 2-algebra $\mathfrak{s l}_{2}(K)$ is not simple, since

$$
\mathfrak{s l}_{2}(K)^{(1)}=\left[\mathfrak{s l}_{2}(K), \mathfrak{s l}_{2}(K)\right]=\operatorname{span}\left\{h_{1}\right\}
$$

and therefore $\mathfrak{s l}_{2}(K)^{(1)}$ is a nontrivial ideal of $\mathfrak{s l}_{2}(K)$. In the next theorem we consider the case where $n \geq 2$.

Theorem 2.2. The special Lie algebra $\mathfrak{s l}_{n+1}(K)$ has the following properties:

(1) $\left(\mathfrak{s l}_{n+1}(K),[2]\right)$ is a Lie 2-algebra.

(2) If $n \geq 2$ and $(n+1) \not \equiv 0 \bmod 2$, then $\mathfrak{s l}_{n+1}(K)$ is a simple Lie 2-algebra.

(3) $\mathfrak{p s l}_{2 n}(K):=\mathfrak{s l}_{2 n}(K) / \mathfrak{z}\left(\mathfrak{g l}_{2 n}(K)\right), n \geq 2$, is a simple Lie 2-algebra.

Proof. In order to prove (1), it is enough to see that $\mathfrak{s l}_{n}(K)$ is closed by the 2-map $[2]: \mathfrak{s l}_{n+1}(K) \rightarrow \mathfrak{s l}_{n+1}(K)$. But this is an immediate consequence of the fact that $\operatorname{tr}\left(A^{2}\right)=\operatorname{tr}(A)^{2}$, for all $A \in \mathfrak{s l}_{n+1}(K)$. 
Let us prove (2). Firstly, we show that if $(n+1) \not \equiv 0 \bmod 2$, then

$$
\mathfrak{g l}_{n+1}(K)=\mathfrak{s l}_{n+1}(K) \oplus \mathfrak{z}\left(\mathfrak{g l}_{n+1}(K)\right) .
$$

Indeed, if $A \in \mathfrak{g l}_{n+1}(K)$ and $\operatorname{tr}(A)=\lambda$, then

$$
A=\left(A-\frac{\lambda}{n+1} I_{n+1}\right)+\frac{\lambda}{n+1} I_{n+1}
$$

where $\left(A-\frac{\lambda}{n+1} I_{n+1}\right) \in \mathfrak{s l}_{n+1}(K)$ and $\frac{\lambda}{n+1} I_{n+1} \in \mathfrak{z}\left(\mathfrak{g l}_{n+1}(K)\right)$. If $A \in \mathfrak{s l}_{n+1}(K) \cap$ $\mathfrak{z}\left(\mathfrak{g l}_{n+1}(K)\right)$, then $A=\lambda I_{n+1}$ and $\operatorname{tr}(A)=(n+1) \lambda=0$. Since $n+1$ is not a multiple of 2 , we have $\lambda=0$. Therefore, $\mathfrak{s l}_{n+1}(K) \cap \mathfrak{z}\left(\mathfrak{g l}_{n+1}(K)\right)=\{0\}$. Now, let $I$ be an ideal of $\mathfrak{s l}_{n+1}(K)$. Then

$$
\begin{aligned}
{\left[\mathfrak{g l}_{n}(K), I\right] } & =\left[\mathfrak{s l}_{n+1}(K) \oplus \mathfrak{z}\left(\mathfrak{g l}_{n+1}(K)\right), I\right] \\
& =\left[\mathfrak{s l}_{n+1}(K), I\right]+\left[\mathfrak{z}\left(\mathfrak{g l}_{n+1}(K)\right), I\right] \\
& \subseteq I+0=I .
\end{aligned}
$$

Therefore, $I$ is also an ideal of $\mathfrak{g l}_{n}(K)$. However, the only ideals of $\mathfrak{g l}_{n}(K)$ contained in $\mathfrak{s l}_{n+1}(K)$ are $\{0\}$ and $\mathfrak{s l}_{n+1}(K)$ (see [6]). Then, $I=\{0\}$ or $I=\mathfrak{s l}_{n+1}(K)$. Hence, $\mathfrak{s l}_{n+1}(K)$ is a simple Lie 2-algebra.

We now prove $(3)$. If $(n+1) \equiv 0 \bmod 2$, then $\mathfrak{z}\left(\mathfrak{g l}_{n+1}(K)\right) \subseteq \mathfrak{s l}_{n+1}(K)$ is an ideal of $\mathfrak{s l}_{n+1}(K)$. Therefore, $\mathfrak{s l}_{n+1}(K) / \mathfrak{z}\left(\mathfrak{g l}_{n+1}(K)\right)$ is a Lie 2-algebra with 2-map given by

$$
\left(x+\mathfrak{z}\left(\mathfrak{g l}_{n+1}(K)\right)\right)^{[2]}:=x^{[2]}+\mathfrak{z}\left(\mathfrak{g l}_{n+1}(K)\right), \quad \text { for all } x \in \mathfrak{s l}_{n+1}(K) .
$$

Now, if $J$ is another ideal of $\mathfrak{s l}_{n+1}(K) / \mathfrak{z}\left(\mathfrak{g l}_{n+1}(K)\right)$, then $J=I / \mathfrak{z}\left(\mathfrak{g l}_{n+1}(K)\right)$, where $I$ is an ideal of $\mathfrak{s l}_{n+1}(K)$ and $\mathfrak{z}\left(\mathfrak{g l}_{n+1}(K)\right) \subseteq I$. Suppose that $I \neq \mathfrak{z}\left(\mathfrak{g l}_{n+1}(K)\right)$. Then, by direct computations, we find that $e_{k l} \in I$, with $k \neq l$, and by using the identities

$$
\begin{aligned}
{\left[e_{k l}, e_{l k}\right] } & :=e_{k k}+e_{l l}, \quad k \neq l ; \\
{\left[e_{k+1, l}, e_{l, k+1}\right] } & :=e_{k+1, k+1}+e_{l l}, \quad k \neq l,
\end{aligned}
$$

we obtain $h_{k}:=e_{k, k}+e_{k+1, k+1} \in I$ for all $k=1,2, \ldots, n$. Therefore, $I=\mathfrak{s l}_{n+1}(K)$, and $\mathfrak{s l}_{n+1}(K) / \mathfrak{z}\left(\mathfrak{g l}_{n+1}(K)\right)$ is a simple Lie 2-algebra.

Recall some well-known facts about quadratic forms over an algebraically closed field of characteristic 2 and their corresponding Lie algebras. Let $V$ be an $n$ dimensional vector space over $K$, and let $b: V \times V \rightarrow K$ be a non-degenerate symmetric bilinear form. This means that $b(x, y)=b(y, x)$, for all $x, y \in V$, and $b(x, V)=0$ implies $x=0$. A non-degenerate symmetric bilinear form $b$ is called symplectic if $b(x, x)=0$ for all $x \in V$. Otherwise, it is called an orthogonal bilinear form. 


\section{Lie 2-Algebras $(\mathfrak{g}(V, b),[2])$ With $b$ A SYMPleCtiC BILINEAR FORM}

In this section we study the simplicity of Lie 2-algebras which preserve a bilinear symplectic form over $K$.

Let $b: V \times V \rightarrow K$ be a symplectic bilinear form. From Example 1.3, we have that $\mathfrak{g}(V, b)$ is a Lie 2-algebra. We denote this algebra by $\mathfrak{s p}(V, b)$, and call it the symplectic Lie 2-algebra. In [8, Theorem 19] it is shown that the dimension of $V$ is even, that is, $n=2 m$, and there exists a basis $\beta$ of $V$ in which $b$ has Gram matrix

$$
J_{2 m}:=\left(\begin{array}{cc}
0 & I_{m} \\
-I_{m} & 0
\end{array}\right) .
$$

The Lie 2-algebra $\mathfrak{s p}(V, b)$ is isomorphic to the Lie 2-algebra

$$
\mathfrak{s p}_{2 m}(K):=\mathfrak{g}\left(J_{2 m}\right)=\left\{\left(\begin{array}{cc}
a & b \\
c & a^{T}
\end{array}\right): a, b, c \in \mathfrak{g l}_{m}(K) \text {, with } b, c \text { symmetric }\right\},
$$

which has dimension $2 m^{2}+m$ and a basis consisting of the following elements:

$$
\begin{array}{rlrl}
d_{i} & :=e_{i i}+e_{m+i, m+i}, & & 1 \leq i \leq m ; \\
a_{i j} & :=e_{i j}+e_{m+j, m+i}, & & 1 \leq i, j \leq m, i \neq j ; \\
b_{i j} & :=e_{i, j+m}+e_{j, i+m}, & & 1 \leq i<j \leq m, i \neq j ; \\
b_{i} & :=e_{i, i+m}, & 1 \leq i \leq m ; \\
c_{i j} & :=e_{i+m, j}+e_{j+m, i}, & 1 \leq i<j \leq m, i \neq j ; \\
c_{i} & :=e_{i+m, i}, & 1 \leq i \leq m .
\end{array}
$$

\begin{tabular}{|c|c|c|c|c|c|c|}
\hline & $d_{i}$ & $a_{i j}$ & $b_{i j}$ & $c_{i j}$ & $b_{i}$ & $c_{i}$ \\
\hline$d_{i}$ & $d_{i}$ & $a_{i j}$ & $b_{i j}$ & $c_{i j}$ & 0 & 0 \\
\hline$a_{i j}$ & $a_{i j}$ & 0 & 0 & 0 & 0 & $a_{i j}$ \\
\hline$b_{i j}$ & $b_{i j}$ & 0 & 0 & $d_{i}+d_{j}$ & 0 & $a_{i j}$ \\
\hline$c_{i j}$ & $c_{i j}$ & 0 & $d_{i}+d_{j}$ & 0 & $a_{i j}$ & 0 \\
\hline$b_{i}$ & 0 & 0 & 0 & $a_{i j}$ & 0 & $d_{i}$ \\
\hline$c_{i}$ & 0 & $c_{i j}$ & $a_{i j}$ & 0 & $d_{i}$ & 0 \\
\hline
\end{tabular}

The Lie bracket of $\mathfrak{s p}_{2 m}(K)$ is given in Table 1, where the elements of the diagonal are results of the 2-map in the elements of their rows and corresponding columns.

We now calculate the derived algebras of $\mathfrak{s p}_{2 m}(K)$, and then we show that the second derived algebra is a simple Lie 2-algebra whenever 2 does not divide $m$ and $m \geq 3$.

Remark 3.1. For $m=1$, we have $\mathfrak{s p}_{2}(K)=\operatorname{span}\left\{d_{1}, b_{12}, c_{21}\right\}=\mathfrak{s l}_{2}(K)$. Then

$$
\mathfrak{s p}_{2}(K)^{(1)}=\operatorname{span}\left\{h_{1}\right\}=\mathfrak{z}\left(\mathfrak{g l}_{2}(K)\right) \quad \text { and } \quad \mathfrak{s p}_{2}(K)^{(2)}=\{0\} \text {, }
$$


and for $m=2$, we have $\mathfrak{s p}_{4}(K)=\operatorname{span}\left\{d_{1}, d_{2}, a_{12}, a_{21}, b_{12}, b_{1}, b_{2}, c_{12}, c_{1}, c_{2}\right\}$. By direct computations, we obtain

$$
\begin{aligned}
& \mathfrak{s p}_{4}(K)^{(1)}=\operatorname{span}\left\{d_{1}, d_{2}, a_{12}, a_{21}, b_{12}, c_{12}\right\}, \\
& \mathfrak{s p}_{4}(K)^{(2)}=\operatorname{span}\left\{d_{1}+d_{2}, a_{12}, a_{21}, b_{12}, c_{12}\right\}, \\
& \mathfrak{s p}_{4}(K)^{(3)}=\mathfrak{z}\left(\mathfrak{g l}_{4}(K)\right), \\
& \mathfrak{s p}_{4}(K)^{(4)}=\{0\} .
\end{aligned}
$$

Therefore, if $m=1,2$, then $\mathfrak{s p}_{2 m}(K)$ is a solvable Lie 2-algebra.

Lemma 3.2. If $m \geq 3$, then

(1) $\mathfrak{s p}_{2 m}(K)^{(1)}=\left\{\left(\begin{array}{cc}a & b \\ c & a^{T}\end{array}\right): b, c \in \operatorname{Alt}_{m}(K), a \in \mathfrak{g l}_{m}(K)\right\}$,

(2) $\mathfrak{s p}_{2 m}(K)^{(2)}=\left\{\left(\begin{array}{cc}a & b \\ c & a^{T}\end{array}\right): b, c \in \operatorname{Alt}_{m}(K), \operatorname{tr}(a)=0\right\}$,

(3) $\mathfrak{s p}_{2 m}(K)^{(3)}=\mathfrak{s p}_{2 m}(K)^{(2)}$,

where $\operatorname{Alt}_{m}(K)$ is the set of alternating $(m \times m)$-matrices with entries in $K$.

Proof. To prove (1), we set $\mathfrak{g}_{1}:=\left\{\left(\begin{array}{cc}a & b \\ c & a^{T}\end{array}\right): b, c \in \operatorname{Alt}_{m}(K)\right\}$. By direct calculations, we show that $\mathfrak{s p}_{2 m}(K)^{(1)} \subseteq \mathfrak{g}_{1}$. Now, we show that $\mathfrak{g}_{1} \subseteq \mathfrak{s p}_{2 m}(K)^{(1)}$. Given $a=\left(x_{i j}\right) \in \mathfrak{g l}_{m}(K)$, we have

$$
\left(\begin{array}{cc}
a & 0 \\
0 & a^{T}
\end{array}\right)=\sum_{i=1}^{m} x_{i i}\left[b_{i}, c_{i}\right]+\sum_{i \neq j}^{m} x_{i j}\left[b_{i}, c_{i j}\right] \in \mathfrak{s p}_{2 m}(K)^{(1)} .
$$

Let us consider the linear map $\varphi: \mathfrak{g l}_{m}(K) \rightarrow \operatorname{Alt}_{m}(K)$ given by $a \mapsto a+a^{T}$. Since $\operatorname{Ker}(\varphi)=\left\{a \in \mathfrak{g l}_{m}(K): a\right.$ is symmetric $\}$ and

$$
\operatorname{dim}_{K}(\operatorname{Im}(\varphi))=m^{2}-\frac{m(m+1)}{2}=\frac{m(m-1)}{2}=\operatorname{dim}_{K}\left(\operatorname{Alt}_{m}(K)\right),
$$

we conclude that $\operatorname{Im}(\varphi)=\operatorname{Alt}_{m}(K)$. That is, $\varphi$ is a surjective map. Then, given $b \in \operatorname{Alt}_{m}(K)$ there exists $a \in \mathfrak{g l}_{m}(K)$ such that $a+a^{T}=b$. Hence,

$$
\left(\begin{array}{ll}
0 & b \\
0 & 0
\end{array}\right)=\left(\begin{array}{cc}
0 & a+a^{T} \\
0 & 0
\end{array}\right)=\left[\left(\begin{array}{cc}
a & 0 \\
0 & a^{T}
\end{array}\right),\left(\begin{array}{cc}
0 & I \\
0 & 0
\end{array}\right)\right] \in \mathfrak{s p}_{2 m}(K)^{(1)} \text {. }
$$

Similarly, we prove that $\left(\begin{array}{cc}0 & 0 \\ c & 0\end{array}\right) \in \mathfrak{s p}_{2 m}(K)^{(1)}$. Therefore, $\mathfrak{g}_{1} \subseteq \mathfrak{s p}_{2 m}(K)^{(1)}$.

To prove (2), let $\mathfrak{g}_{2}=\left\{\left(\begin{array}{cc}a & b \\ c & a^{T}\end{array}\right): b, c \in \operatorname{Alt}_{m}(K), \operatorname{tr}(a)=0\right\}$. We will prove that $\mathfrak{s p}_{2 m}(K)^{(2)}=\mathfrak{g}_{2}$. From the description of $\mathfrak{s p}_{2 m}(K)^{(1)}$ in (1), we deduce that the Lie algebra $\mathfrak{s p}_{2 m}(K)^{(1)}$ is generated by $a_{i j}, b_{i j}, c_{i j}$, and $d_{i}$ for $1 \leq i, j \leq m$. Therefore, $\mathfrak{s p}_{2 m}(K)^{(2)}=\left[\mathfrak{s p}_{2 m}(K)^{(1)}, \mathfrak{s p}_{2 m}(K)^{(1)}\right]$ is generated by $a_{i j}, b_{i j}, c_{i j}$, and $d_{i}+d_{j}$ for $1 \leq i, j \leq m$. Since all of these elements belong to $\mathfrak{g}_{2}$, we conclude that $\mathfrak{s p}_{2 m}(K)^{(2)} \subseteq \mathfrak{g}_{2}$. The other inclusion is established reasoning as in the proof of (1). 
Finally, we prove (3). In the proof of (2), it is shown that $\mathfrak{s p}_{2 m}(K)^{(2)}$ is generated by $d_{i}+d_{j}$ for $1 \leq i, j \leq m$. Therefore,

$$
\mathfrak{s p}_{2 m}(K)^{(3)}=\operatorname{span}\left\{[x, y]: x, y \in\left\{a_{i j}, b_{i j}, c_{i j}, d_{i}+d_{j}: 1 \leq i, j \leq m\right\}\right\} .
$$

From Table 1, we conclude that

$$
\mathfrak{s p}_{2 m}(K)^{(3)}=\operatorname{span}\left\{a_{i j}, b_{i j}, c_{i j}, d_{i}+d_{j}: 1 \leq i, j \leq m\right\}=\mathfrak{s p}_{2 m}(K)^{(2)} .
$$

Lemma 3.3. Let $I$ be a nontrivial ideal of $\mathfrak{s p}_{2 m}(K)^{(2)}$. Then $c_{i j}, b_{i j} \notin I$, for all $i, j$.

Proof. Let $1 \leq i \neq j \leq n$ be fixed. If $c_{i j} \in I$, then for all $k \neq j$ the relations $\left[c_{i j}, b_{i j}\right]=d_{i}+d_{j},\left[d_{i}+d_{j}, a_{i k}\right]=a_{i k},\left[d_{i}+d_{j}, c_{i k}\right]=c_{i k}$, and $\left[d_{i}+d_{j}, b_{i k}\right]=b_{i k}$ imply that $a_{i k}, b_{i k}, d_{i}+d_{k}$ belong to $I$ for all $1 \leq k \leq m$. Since $I$ is an ideal of $\mathfrak{s p}_{2 m}(K)^{(2)}$, for all $l, k$ with $l \neq k$ we have that $\left[a_{i l}, c_{i k}\right]=c_{l k},\left[b_{i l}, c_{i k}\right]=a_{l k}$, and $\left[d_{i}+d_{l}, b_{l k}\right]=b_{l k}$ belong to $I$. Therefore, $I=\mathfrak{s p}_{2 m}(K)^{(2)}$, which is a contradiction. Similarly, if we suppose that $b_{i j} \in I$, we arrive at a contradiction. Hence, $c_{i j}, b_{i j} \notin I$ for all $i, j$.

Theorem 3.4. Let $b: V \times V \rightarrow K$ be a symplectic bilinear form and let $\mathfrak{s p}(V, b)$ be the sympletic Lie algebra associated to $b$. Suppose that $\operatorname{dim}_{K}(V)=n>4$. Then

(1) $\left(\mathfrak{s p}(V, b)^{(2)}, 2\right)$ is a Lie 2-algebra.

(2) If $4 \mid n$, then $\mathfrak{s p}(V, b)^{(2)} / \mathfrak{z}(\mathfrak{g l}(V))$ is simple.

(3) If $4 \nmid n$, then $\mathfrak{s p}(V, b)^{(2)}$ is simple.

Proof. The proof of (1) is completely based on the definition of the algebra $\operatorname{Alt}_{m}(K)$, so we omit it. Let us prove (2). If $4 \mid 2 m$, then $\mathfrak{z}\left(\mathfrak{g l}_{2 m}(K)\right) \subseteq \mathfrak{s p}_{2 m}(K)^{(2)}$ is an ideal of $\mathfrak{s p}_{2 m}(K)^{(2)}$. Let $J$ be an ideal of $\mathfrak{s p}_{2 m}(K)^{(2)} / \mathfrak{z}\left(\mathfrak{g l}_{2 m}(K)\right)$; then $J=I / \mathfrak{z}\left(\mathfrak{g l}_{2 m}(K)\right)$, where $I$ is an ideal of $\mathfrak{s p}_{2 m}(K)^{(2)}$ and $\mathfrak{z}\left(\mathfrak{g l}_{2 m}(K)\right) \subseteq I$. Suppose that $I \neq \mathfrak{s p}_{2 m}(K)^{(2)}$. By Lemma 3.3 we have $c_{i j}, b_{i j} \notin I$; therefore, given $\alpha \in I$ there exists $a=\left(a_{i j}\right) \in \mathfrak{g}_{m}(K)$ with $\operatorname{tr}(a)=0$ such that

$$
\alpha=\left(\begin{array}{cc}
a & 0 \\
0 & a^{T}
\end{array}\right) \text {. }
$$

Now, since $I$ is an ideal of $\mathfrak{s p}_{2 m}(K)^{(2)}$, we get

$$
[\alpha, X] \in I, \quad \text { for all } X \in \mathfrak{s p}_{2 m}(K)^{(2)} \text {. }
$$

In particular, for

$$
X=\left(\begin{array}{ll}
0 & b \\
0 & 0
\end{array}\right),
$$

where $b:=e_{i j}+e_{j i} \in \operatorname{Alt}_{m}(K)$, we have $a_{i j}=0$ for $i \neq j$ and $a_{i i}=a_{11}$ for all $i$. Then $\alpha=a_{i i} I_{2 m} \in \mathfrak{z}\left(\mathfrak{g l}_{2 m}(K)\right)$. Hence, $I=\mathfrak{z}\left(\mathfrak{g l}_{2 m}(K)\right)$ and $\mathfrak{s p}_{2 m}(K)^{2} / \mathfrak{z}\left(\mathfrak{g l}_{2 m}(K)\right)$ is simple.

To complete the proof, we prove (3). Let $I$ be an ideal of $\mathfrak{s p}_{2 m}(K)^{(2)}, I \neq$ $\mathfrak{s p}_{2 m}(K)^{(2)}$. Reasoning as in the proof of item (2), we get $a=\lambda I_{m}$ with $m$ odd. As $\operatorname{tr}(a)=0$, we have $\lambda=0$. Then $\alpha=0$ and $I=\{0\}$. Therefore, $\mathfrak{s p}_{2 m}(K)^{(2)}$ is simple. 


\section{Lie 2-Algebras $(\mathfrak{g}(V, b),[2])$ With $b$ AN ORThOGONAL BILINEAR FORM}

In this section we show that the Lie algebra which preserves the orthogonal linear form over $K$ is not a Lie 2-subalgebra of $\mathfrak{g l}_{n}(K)$.

Suppose that $b: V \times V \rightarrow K$ is an orthogonal bilinear form, and let $\mathfrak{o}(V, b)$ be the Lie 2-algebra associated to $b$.

In [8, Theorem 20] it is shown that there exists a basis $\beta$ of $V$ in which $b$ has Gram matrix $D=\operatorname{diag}\left(d_{1}, d_{2}, \ldots, d_{n}\right)$, where $0 \neq d_{i} \in K$ for all $i$; therefore, the matricial algebra $\mathfrak{g}(D)$ corresponding to the Lie 2-algebra $\mathfrak{o}(V, b)$ is given by

$$
\mathfrak{g}(D):=\left\{A \in \mathfrak{g l}_{n}(K): d_{i} a_{i j}=d_{j} a_{j i}, 1 \leq i, j \leq n\right\} .
$$

Since $K$ is an algebraically closed field, we have that $K^{2}=K$, that is, every element of $K$ is a square. This fact implies that we can take the diagonal matrix $D$ as the identity matrix $I_{n}$. So,

$$
\mathfrak{o}_{n}(K):=\mathfrak{g}\left(I_{n}\right)=\left\{A \in \mathfrak{g l}_{n}(K): A \text { is symmetric }\right\}
$$

is a Lie 2-algebra with basis $\left\{e_{i i}\right\} \cup\left\{\bar{e}_{i j}:=e_{i j}+e_{j i}\right\}, 1 \leq i<j \leq n$, and whose Lie bracket is given by

$$
\begin{aligned}
{\left[\bar{e}_{i i}, \bar{e}_{i j}\right] } & =\bar{e}_{i j}, \quad 1 \leq i<j \leq n . \\
{\left[\bar{e}_{i i}, \bar{e}_{k k}\right] } & =0, \quad i \neq k . \\
{\left[\bar{e}_{i j}, \bar{e}_{k l}\right] } & =\delta_{i k} \bar{e}_{j l}+\delta_{i l} \bar{e}_{k j}+\delta_{j k} \bar{e}_{i l}+\delta_{j l} \bar{e}_{i k}, \quad \text { for all } i<j, k<l .
\end{aligned}
$$

Moreover, $\bar{e}_{i j}^{2}=e_{i i}+e_{j j}$, and $\operatorname{dim}_{K}\left(\mathfrak{o}_{n}(K)\right)=\frac{n(n+1)}{2}$.

Lemma 4.1. $\mathfrak{o}_{n}(K)^{(1)}=\operatorname{Alt}_{n}(K)$ and $\operatorname{dim}_{K}\left(\mathfrak{o}_{n}(K)^{(1)}\right)=\frac{n(n-1)}{2}$.

Proof. Based on the definition of $\mathfrak{o}_{n}(K)$ it is very easy to prove that $\mathfrak{o}_{n}(K)^{(1)} \subseteq$ $\operatorname{Alt}_{n}(K)$. Now, the matrices $\bar{e}_{i j}:=e_{i j}+e_{j i}$, where $1 \leq i<j \leq n$, form a basis of $\operatorname{Alt}_{n}(K)$, and $\bar{e}_{i j}=\left[\bar{e}_{i k}, \bar{e}_{k j}\right] \in \mathfrak{o}_{n}(K)^{(1)}$ for all $i, j$, Then, $\mathfrak{o}_{n}(K)^{(1)}=\operatorname{Alt}_{n}(K)$ and $\operatorname{dim}_{K}\left(\mathfrak{o}_{n}(K)^{(1)}\right)=\operatorname{dim}_{K}\left(\mathfrak{o}_{n}(K)\right)-n=\frac{n(n-1)}{2}$.

Remark 4.2. A direct calculation shows that $\bar{e}_{i j}^{2}=e_{i i}+e_{j j}$, for $i \neq j$. Then, $\bar{e}_{i j}^{2} \notin \mathfrak{o}_{n}(K)^{(1)}$. Therefore, $\mathfrak{o}_{n}(K)^{(1)}$ is not a Lie 2 -algebra with respect to the 2-map [2] : $\mathfrak{o}_{n}(K) \rightarrow \mathfrak{o}_{n}(K)$ defined by $a \mapsto a^{2}$.

\section{Classical type simple Lie 2-Algebras and their toral Rank}

W. Killing and E. Cartan showed that all simple Lie algebras over an algebraically closed field of characteristic zero is isomorphic to one of the classical Lie algebras $A_{n}(n \geq 1), B_{n}(n \geq 2), C_{n}(n \geq 3), D_{n}(n \geq 4)$, or to the exceptional Lie algebras $\mathfrak{g}_{2}, \mathfrak{f}_{4}, \mathfrak{e}_{6}, \mathfrak{e}_{7}, \mathfrak{e}_{8}$ (see [5]). But in characteristic 2 , it seems that many new phenomena arise; for instance, these algebras are not necessarily simple, or some of them are isomorphic, and therefore, the classification of simple Lie algebras in characteristic 2 will differ from the classification of such algebras in characteristics 0 and $p \geq 5$. In this section, we calculate the toral rank of the simple Lie 2-algebra of the classical type and we conclude that there are no classical type simple Lie 
2-algebras of odd toral rank. In particular, there are no classical type simple Lie 2-algebras of toral rank 3 .

Definition 5.1. Given an irreducible root system of type $X_{l}$ and its corresponding Chevalley algebra $\mathfrak{g}\left(X_{l}, K\right)$ over the field $K$, the quotient

$$
\overline{\mathfrak{g}\left(X_{l}, K\right)}:=\mathfrak{g}\left(X_{l}, K\right) / \mathfrak{z}\left(\mathfrak{g}\left(X_{l}, K\right)\right),
$$

where $\mathfrak{z}\left(\mathfrak{g}\left(X_{l}, K\right)\right)$ is the center of $\mathfrak{g}\left(X_{l}, K\right)$, is usually called the classical Lie algebra of type $X_{l}$.

Remark 5.2. This definition is exactly the same as Steinberg's [11, but Steinberg excluded some types of characteristic 2 and 3 .

The simplicity of the classical type Lie algebras in characteristic 2 has been determined by Hogeweij in [4], as indicated in the following proposition.

Proposition 5.3. Suppose that $X_{l}$ is a Lie algebra which is not of type $A_{1}, B_{l}$, $C_{l}$, or $\mathfrak{f}_{4}$. Then $\overline{\mathfrak{g}\left(X_{l}, K\right)}$ is a simple Lie 2-algebra.

We can summarize Proposition 5.3, Theorem 2.2, and Theorem 3.4 in the following corollary.

Corollary 5.4. The classical type simple Lie 2-algebras are:

(1) Type $A_{l}$ :

$$
\begin{aligned}
& \overline{\mathfrak{g}\left(A_{l}, K\right)} \simeq \mathfrak{s l}_{l+1}(K), \\
& \text { if } 2 \nmid(l+1), l>1 \text {; } \\
& \overline{\mathfrak{g}\left(A_{l}, K\right)} \simeq \mathfrak{p s l}_{l+1}(K), \\
& \text { if } 2 \mid(l+1), l>1 \text {. }
\end{aligned}
$$

(2) Type $D_{l}$ :

$$
\begin{aligned}
& \overline{\mathfrak{g}\left(D_{l}, K\right)} \simeq \mathfrak{s p}_{2 l}(K)^{(2)}, \\
& \text { if } l \text { is odd; } \\
& \overline{\mathfrak{g}\left(D_{l}, K\right)} \simeq \mathfrak{s p}_{2 l}(K)^{(2)} / \mathfrak{z}\left(\mathfrak{g l}_{2 l}(K)\right), \\
& \text { if } l \text { is even. }
\end{aligned}
$$

(3) Type $\mathfrak{g}_{2}$ :

$$
\overline{\mathfrak{g}\left(\mathfrak{g}_{2}, K\right)}=\mathfrak{g}\left(\mathfrak{g}_{2}, K\right) .
$$

(4) Type $\mathfrak{e}_{6}$ :

$$
\overline{\mathfrak{g}\left(\mathfrak{e}_{6}, K\right)}=\mathfrak{g}\left(\mathfrak{e}_{6}, K\right)
$$

(5) Type $\mathfrak{e}_{7}$ :

$$
\overline{\mathfrak{g}\left(\mathfrak{e}_{7}, K\right)}
$$

(6) Type $\mathfrak{e}_{8}$ :

$$
\overline{\mathfrak{g}\left(\mathfrak{e}_{8}, K\right)}=\mathfrak{g}\left(\mathfrak{e}_{8}, K\right) .
$$

Theorem 5.5. Let $\mathfrak{g}$ be a classical type simple Lie 2-algebra and let $\mathfrak{h}$ be a Cartan subalgebra of $\mathfrak{g}$. Then

$$
M T(\mathfrak{g})=\operatorname{dim}_{K}(\mathfrak{h}) .
$$


Proof. Let $\mathfrak{g}$ be a classical type simple Lie 2-algebra. Then from Corollary 5.4 it follows that $\mathfrak{g}=\overline{\mathfrak{g}\left(X_{l}, K\right)}$ with $X_{l} \neq A_{1}, B_{l}, C_{l}, \mathfrak{f}_{4}$. Hence, any quotient of the form

$$
\overline{\mathfrak{h}\left(X_{l}, K\right)}=\mathfrak{h}\left(X_{l}, K\right) / \mathfrak{z}\left(\mathfrak{g}\left(X_{l}, K\right)\right),
$$

where $\mathfrak{h}\left(X_{l}, K\right)$ is a Cartan subalgebra of the Chevalley $K$-algebra $\mathfrak{g}\left(X_{l}, K\right)$, is a Cartan subalgebra of $\mathfrak{g}$. Since $\mathfrak{h}\left(X_{l}, K\right)=\operatorname{span}\left\{h_{i} \otimes 1: h_{i} \in \mathfrak{h}_{X_{l}}\right\}$ and $\mathfrak{h}_{X_{l}}$ is the subalgebra of diagonal matrices of $\mathfrak{s l}_{l+1}(K)$, we obtain $\left(h_{i} \otimes 1\right)^{[2]}=h_{i} \otimes 1$, for each $h_{i} \in \mathfrak{h}_{X_{l}}$. Thus, the equality $\left(\left[h_{i} \otimes 1\right]\right)^{[2]}=\left[h_{i} \otimes 1\right]$ modulo $\mathfrak{z}\left(\mathfrak{g}\left(X_{l}, K\right)\right)$ implies that $\overline{\mathfrak{h}\left(X_{l}, K\right)} \subseteq T\left(\overline{\mathfrak{h}\left(X_{l}, K\right)}\right)$, and as $T\left(\overline{\mathfrak{h}\left(X_{l}, K\right)}\right) \subseteq \overline{\mathfrak{h}\left(X_{l}, K\right)}$, we have that $\overline{\mathfrak{h}\left(X_{l}, K\right)}=T\left(\overline{\mathfrak{h}\left(X_{l}, K\right)}\right)$. Since any pair of Cartan Lie subalgebras of a finitedimensional classical type Lie algebra $\mathfrak{g}$ over $K$ are conjugate (see [9]), there exists an automorphism $\sigma \in \operatorname{Aut}(\mathfrak{g})$ such that $\mathfrak{h}=\sigma\left(\overline{\mathfrak{h}\left(X_{l}, K\right)}\right)$. Then, from [1, Lemma 5] we obtain

$$
T(\mathfrak{h})=\sigma\left(T\left(\overline{\mathfrak{h}\left(X_{l}, K\right)}\right)\right)=\sigma\left(\overline{\mathfrak{h}\left(X_{l}, K\right)}\right)=\mathfrak{h} .
$$

Then any Cartan subalgebra of a simple Lie 2-algebra $\mathfrak{g}$ of classical type is a maximal torus in $\mathfrak{g}$, and hence

$$
M T(\mathfrak{g})=\operatorname{dim}_{K}(\mathfrak{h}) .
$$

The following is a direct consequence of Theorem 5.5

Corollary 5.6. The toral rank of the classical type simple Lie 2-algebras is:

(1) $M T\left(A_{l}\right)=l, \quad$ if $2 \nmid(l+1), l>1$;

(2) $M T\left(A_{l}\right)=l-1, \quad$ if $2 \mid(l+1), l>1$;

(3) $M T\left(D_{l}\right)=l-1, \quad$ if $l$ is odd, $l \geq 3$;

(4) $M T\left(D_{l}\right)=l-2, \quad$ if $l$ is even, $l \geq 3$;

(5) $M T\left(\mathfrak{g}_{2}\right)=2$;

(6) $M T\left(\mathfrak{e}_{6}\right)=6$;

(7) $M T\left(\mathfrak{e}_{7}\right)=6$;

(8) $M T\left(\mathfrak{e}_{8}\right)=8$.

From this corollary, the next result follows.

Theorem 5.7. There are no classical type simple Lie 2-algebras of odd toral rank.

\section{A (CONTRAgredient) Simple Lie 2-Algebra of Dimension 34 AND TORAL RANK 4}

In this section we show that the contragredient Lie 2-algebra $G\left(F_{4, a}\right)$ constructed by V. Kac and V. Veŭsfeĭler (see [13]) has toral rank 4, and we obtain the Cartan decomposition of this algebra.

Definition 6.1. Given an $(n \times n)$-matrix $A=\left(a_{i j}\right)$ with elements in $K$, we denote by $\widetilde{G}(A)$ the Lie algebra determined by the system of generators $e_{i}, f_{i}, h_{i}, i=$ $1, \ldots, n$, and the system of relations

$$
\left[e_{i}, f_{j}\right]=\delta_{i j} h_{j}, \quad\left[h_{i}, h_{j}\right]=0, \quad\left[h_{i}, e_{j}\right]=a_{i j} e_{j}, \quad\left[h_{i}, f_{j}\right]=-a_{i j} f_{j},
$$


for $1 \leq i, j \leq n$. We set $\operatorname{deg} e_{i}=1, \operatorname{deg} f_{i}=-1$, and $\operatorname{deg} h_{i}=0, i=1,2, \ldots, n$. Thus, the algebra $\widetilde{G}(A)$ becomes a graded Lie algebra, $\widetilde{G}(A)=\bigoplus_{i \in \mathbb{Z}} \widetilde{G}_{i}$. Let $J(A)$ be a maximal homogeneous ideal in $\widetilde{G}(A)$ such that $J(A) \cap\left(\widetilde{G}_{-1} \oplus \widetilde{G}_{0} \oplus \widetilde{G}_{1}\right)=0$. The Lie algebra $G(A):=\widetilde{G}(A) / J(A)$ is called a contragredient Lie algebra and $A$ is its Cartan matrix.

In [13, V. Kac and V. Vĕsfeller considered the algebra

$$
G\left(F_{4, a}\right):=\widetilde{G}\left(F_{4, a}\right) / J\left(F_{4, a}\right),
$$

where

$$
F_{4, a}:=\left(\begin{array}{cccc}
0 & 1 & 0 & 0 \\
a & 0 & 1 & 0 \\
0 & 1 & 0 & 1 \\
0 & 0 & 1 & 0
\end{array}\right),
$$

$a \in K \backslash\{0,1\}$, and $J\left(F_{4, a}\right)$ is the only maximal homogeneous ideal in $\widetilde{G}\left(F_{4, a}\right)$ such that

$$
\left\{\begin{array}{l}
J\left(F_{4, a}\right) \cap \operatorname{span}\left\{h_{1}, h_{2}, h_{3}, h_{4}\right\}=0, \\
J\left(F_{4, a}\right) \cap \operatorname{span}\left\{e_{i}, f_{j}\right\}=0 .
\end{array}\right.
$$

They proved that $G\left(F_{4, a}\right)$ is a simple Lie 2-algebra of dimension 34 with Cartan matrix $F_{4, a}$, with $a \in K \backslash\{0,1\}$ (see [13. Proposition 3.6]). We now prove that this Lie 2-algebra has toral rank 4 and, furthermore, we give its Cartan decomposition.

From (6.1), we conclude that

$$
\mathfrak{h}:=\operatorname{span}\left\{\bar{h}_{i}:=h_{i}+J\left(F_{4, a}\right): i \in I_{4}\right\}
$$

is a Cartan subalgebra of $G\left(F_{a, 4}\right)$. We now explicitly describe the maximal torus $T(\mathfrak{h})$ consisting of toroidal elements in $\mathfrak{h}$.

Since $h_{1}^{[2]} \in \operatorname{span}\left\{h_{1}, h_{2}, h_{3}, h_{4}\right\}$ (Cartan subalgebra of $\widetilde{G}\left(F_{4, a}\right)$ ), we have $h_{1}^{[2]}=$ $\delta_{1} h_{1}+\delta_{2} h_{2}+\delta_{3} h_{3}+\delta_{4} h_{4}$, and by using the relations 6.1) we obtain

$$
0=\left[h_{1},\left[h_{1}, e_{1}\right]\right]=\delta_{1}\left[h_{1}, e_{1}\right]+\delta_{2}\left[h_{2}, e_{1}\right]+\delta_{3}\left[h_{3}, e_{1}\right]+\delta_{4}\left[h_{4}, e_{1}\right]=\delta_{2} a e_{1},
$$

which implies that $\delta_{2}=0$. Similarly, we obtain $\delta_{2}=\delta_{3}=\delta_{4}=0$ and $\delta_{1}=1$. So, $h_{1}^{[2]}=h_{1}$.

We also find $h_{3}^{[2]}=h_{3}, h_{4}^{[2]}=h_{4}$, and $h_{2}^{[2]}=a h_{2}+\bar{a} h_{4}$, where $\bar{a}=a+1$.

Let $t_{2}:=x h_{2}+y h_{4}$, with $x, y \in K$. If the equality $t_{2}^{[2]}=t_{2}$ holds true, then $x, y$ satisfy the system of equations

$$
\left\{\begin{aligned}
a x^{2}+x & =0 \\
\bar{a} x^{2}+y^{2}+y & =0
\end{aligned}\right.
$$

whose solution set is

$$
\left\{(0,0),(0,1),\left(\frac{1}{a}, \frac{1}{a}\right),\left(\frac{1}{a}, \frac{\bar{a}}{a}\right)\right\} .
$$


The first two solutions give $t_{2}=0$ and $t_{2}=h_{4}$, respectively, and with the last two solutions we obtain $t_{2}=\frac{1}{a}\left(h_{2}+h_{4}\right)$ and $t_{2}=\frac{1}{a}\left(h_{2}+\bar{a} h_{4}\right)$. Since $\frac{1}{a}\left(h_{2}+h_{4}\right)=$ $\frac{1}{a}\left(h_{2}+\bar{a} h_{4}\right)-h_{4}$, we have

$$
T(\mathfrak{h}):=\operatorname{span}\left\{\bar{h}_{1}, \bar{h}_{3}, \bar{h}_{4}, \frac{1}{a}\left(h_{2}+h_{4}\right)+J\left(F_{4, a}\right)\right\}
$$

and $\operatorname{dim}_{K}(T(\mathfrak{h}))=4$. This fact shows that $M T\left(G\left(F_{4, a}\right)\right)=4$.

We now find the Cartan decomposition of $G\left(F_{4, a}\right)$ with respect to $T(\mathfrak{h})$. By definition of the ideal $J\left(F_{4, a}\right)$, the elements $e_{i}, f_{i}$, and $h_{i}$, for $1 \leq i \leq 4$, do not belong to $J\left(F_{4, a}\right)$. Therefore, the classes $\bar{e}_{i}=e_{i}+J\left(F_{4, a}\right), \bar{f}_{i}=f_{i}+J\left(F_{4, a}\right)$, and $\bar{h}_{i}=h_{i}+J\left(F_{4, a}\right)$, for $1 \leq i \leq 4$, belong to a basis for $G\left(F_{4, a}\right)$. Now, to complete a basis for $G\left(F_{4, a}\right)$, we consider the product $x y:=[x, y]$. The products $x y$, where $x$ and $y$ are generators of $G\left(F_{4, a}\right)$ and at most one of them does not belong to $\left\{e_{i}, f_{i}: 1 \leq i \leq 4\right\}$, are members of $\operatorname{span}\left\{h_{i}, e_{i}, f_{i}: 1 \leq i \leq 4\right\}$. Thus, the only products of two generators that give us new generators are $e_{i} e_{j}$ and $f_{i} f_{j}$ with $i<j$. So, the elements $\overline{p_{i j}}=e_{i} e_{j}+J\left(F_{4, a}\right)$ and $\overline{q_{i j}}=f_{i} f_{j}+J\left(F_{4, a}\right)$ with $i<j$ are also generators of $G\left(F_{4, a}\right)$, which are linearly independent with $e_{i} e_{j}+J\left(F_{4, a}\right)$ and $f_{i} f_{j}+J\left(F_{4, a}\right)$. Reasoning in a similar way we obtain that the elements $\left(e_{1} e_{2}\right) e_{3}$, $\left(e_{1} e_{2}\right) e_{4},\left(e_{1} e_{3}\right) e_{4},\left(e_{2} e_{3}\right) e_{4},\left(\left(e_{1} e_{2}\right) e_{3}\right) e_{4}$ modulo $J\left(F_{4, a}\right)$ and $\left(f_{1} f_{2}\right) f_{3},\left(f_{1} f_{2}\right) f_{4}$, $\left(f_{1} f_{3}\right) f_{4},\left(f_{2} f_{3}\right) f_{4},\left(\left(f_{1} f_{2}\right) f_{3} f\right)_{4}$ modulo $J\left(F_{4, a}\right)$ complete a basis for $G\left(F_{4, a}\right)$. We denote this basis by $\Phi$.

Next, we calculate the weights for each element of the basis $\Phi$ of $\widetilde{G}\left(F_{4, a}\right)$ with respect to

which are:

$$
\mathfrak{h}_{1}:=\left\{h_{1}, \frac{1}{a}\left(h_{2}+h_{4}\right), h_{3}, h_{4}\right\},
$$

- $\left[e_{1}, h_{1}\right]=a_{11} e_{1}=0 e_{1}$,

$\left[e_{1}, \frac{1}{a}\left(h_{2}+h_{4}\right)\right]=\frac{1}{a}\left(\left[e_{1}, h_{2}\right]+\left[e_{1}, h_{4}\right]\right)=\frac{1}{a}\left(a e_{1}\right)=1 e_{1}$,

$\left[e_{1}, h_{3}\right]=a_{31} e_{1}=0 e_{1}$,

$\left[e_{1}, h_{4}\right]=a_{41} e_{1}=0 e_{1}$.

Then, the weight of $\bar{e}_{1}$ is $\beta:=(0,1,0,0)$.

- $\left[e_{2}, h_{1}\right]=a_{12} e_{2}=1 e_{2}$,

$\left[e_{2}, \frac{1}{a}\left(h_{2}+h_{4}\right)\right]=\frac{1}{a}\left(\left[e_{2}, h_{2}\right]+\left[e_{2}, h_{4}\right]\right)=0 e_{2}$,

$\left[e_{2}, h_{3}\right]=a_{32} e_{2}=1 e_{2}$,

$\left[e_{1}, h_{4}\right]=a_{41} e_{1}=0 e_{1}$.

Then, the weight of $\bar{e}_{2}$ is $\alpha+\gamma:=(1,0,1,0)$.

- $\left[e_{3}, h_{1}\right]=a_{13} e_{3}=0 e_{3}$,

$\left[e_{3}, \frac{1}{a}\left(h_{2}+h_{4}\right)\right]=\frac{1}{a}\left(\left[e_{3}, h_{2}\right]+\left[e_{3}, h_{4}\right]\right)=0 e_{3}$,

$\left[e_{3}, h_{3}\right]=a_{33} e_{3}=0 e_{3}$,

$\left[e_{3}, h_{4}\right]=a_{43} e_{3}=1 e_{3}$.

Then, the weight of $\bar{e}_{3}$ is $\lambda:=(0,0,0,1)$. 
- $\left[e_{4}, h_{1}\right]=a_{14} e_{4}=0 e_{4}$,

$\left[e_{4}, \frac{1}{a}\left(h_{2}+h_{4}\right)\right]=\frac{1}{a}\left(\left[e_{4}, h_{2}\right]+\left[e_{4}, h_{4}\right]\right)=0 e_{4}$,

$\left[e_{4}, h_{3}\right]=a_{34} e_{4}=1 e_{4}$,

$\left[e_{4}, h_{4}\right]=a_{44} e_{4}=0 e_{4}$.

Then, the weight of $\bar{e}_{4}$ is $\gamma:=(0,0,1,0)$.

By the similarity between the definitions of the brackets $\left[h_{i}, f_{j}\right]=a_{i j} f_{j}$ and $\left[h_{i}, e_{j}\right]=a_{i j} e_{j}$, we deduce that $\bar{e}_{i}$ and $\bar{f}_{i}$, for $1 \leq i \leq 4$, have the same weight. On the other hand, by using $\left[\mathfrak{g}_{\xi}, \mathfrak{g}_{\mu}\right] \subseteq \mathfrak{g}_{\xi+\mu}$, we obtain that the remaining elements of $\Phi$ have the weights given in Table 2 , where we use the notation $\overline{p_{i j k}}=\left(e_{i} e_{j}\right) e_{k}+$ $J\left(F_{4, a}\right), \overline{q_{i j k}}=\left(f_{i} f_{j}\right) f_{k}+J\left(F_{4, a}\right), \overline{p_{1234}}=\left(\left(e_{1} e_{2}\right) e_{3}\right) e_{4}+J\left(F_{4, a}\right)$, and $\overline{q_{1234}}=$ $\left(\left(f_{1} f_{2}\right) f_{3}\right) f_{4}+J\left(F_{4, a}\right)$.

\begin{tabular}{lll}
\hline Root space & Basis & Root \\
\hline $\mathfrak{g}_{\beta}$ & $\overline{e_{1}}, \overline{f_{1}}$ & $\beta:=(0,1,0,0)$ \\
\hline $\mathfrak{g}_{\alpha+\gamma}$ & $\overline{e_{2}}, \overline{f_{2}}$ & $\alpha+\gamma:=(1,0,1,0)$ \\
\hline $\mathfrak{g}_{\lambda}$ & $\overline{e_{3}}, \overline{f_{3}}$ & $\lambda:=(0,0,0,1)$ \\
\hline $\mathfrak{g}_{\gamma}$ & $\overline{e_{4}}, \overline{f_{4}}$ & $\gamma:=(0,0,1,0)$ \\
\hline $\mathfrak{g}_{\alpha+\beta+\gamma}$ & $\overline{p_{12}}, \overline{q_{12}}$ & $\alpha+\beta+\gamma:=(1,1,1,0)$ \\
\hline $\mathfrak{g}_{\beta+\lambda}$ & $\overline{p_{13}}, \overline{q_{13}}$ & $\beta+\lambda:=(0,1,0,1)$ \\
\hline $\mathfrak{g}_{\beta+\gamma}$ & $\overline{p_{14}}, \overline{q_{14}}$ & $\beta+\gamma:=(0,1,1,0)$ \\
\hline $\mathfrak{g}_{\alpha+\gamma+\lambda}$ & $\overline{p_{23}}, \overline{q_{23}}$ & $\alpha+\gamma+\lambda:=(1,0,1,1)$ \\
\hline $\mathfrak{g}_{\alpha}$ & $\overline{p_{24}}, \overline{q_{24}}$ & $\alpha:=(1,0,0,0)$ \\
\hline $\mathfrak{g}_{\gamma+\lambda}$ & $\overline{p_{34}}, \overline{q_{34}}$ & $\gamma+\lambda:=(0,0,1,1)$ \\
\hline $\mathfrak{g}_{\alpha+\beta+\gamma+\lambda}$ & $\overline{p_{123}}, \overline{q_{123}}$ & $\alpha+\beta+\gamma+\lambda:=(1,1,1,1)$ \\
\hline $\mathfrak{g}_{\alpha+\beta}$ & $\overline{p_{124}}, \overline{q_{124}}$ & $\alpha+\beta:=(1,1,0,0)$ \\
\hline $\mathfrak{g}_{\beta+\gamma+\lambda}$ & $\overline{p_{134}}, \overline{q_{134}}$ & $\beta+\gamma+\lambda:=(0,1,1,1)$ \\
\hline $\mathfrak{g}_{\alpha+\lambda}$ & $\overline{p_{234}}, \overline{q_{234}}$ & $\alpha+\lambda:=(1,0,0,1)$ \\
\hline $\mathfrak{g}_{\alpha+\beta+\lambda}$ & $\overline{p_{1234}}, \overline{q_{1234}}$ & $\alpha+\beta+\lambda:=(1,1,0,1)$ \\
\hline & TABLE 2. Root spaces of $G\left(F_{4, a}\right)$.
\end{tabular}

Therefore, the Cartan decomposition of $G\left(F_{4, a}\right)$ with respect to $T(\mathfrak{h})$ is

$$
G\left(F_{4, a}\right)=T(\mathfrak{h}) \oplus \bigoplus_{\xi \in G^{*}} \mathfrak{g}_{\xi},
$$

where $G^{*}=G \backslash 0$ and $G:=\langle\alpha, \beta, \gamma, \lambda\rangle$ is an elementary abelian group of order 16 and $\operatorname{dim}_{K}\left(\mathfrak{g}_{\xi}\right)=2$, for all $\xi \in G$. Hence, we have the following theorem. 
Theorem 6.2. The contragredient Lie algebra $G\left(F_{4, a}\right)$ on $K$ with Cartan matrix

$$
F_{4, a}:=\left(\begin{array}{cccc}
0 & 1 & 0 & 0 \\
a & 0 & 1 & 0 \\
0 & 1 & 0 & 1 \\
0 & 0 & 1 & 0
\end{array}\right)
$$

has these properties:

(1) $G\left(F_{4, a}\right)$ is a simple Lie 2-algebra of dimension 34;

(2) $M T\left(G\left(F_{4, a}\right)\right)=4$;

(3) The Cartan decomposition of $G\left(F_{4, a}\right)$ with respect to $T(\mathfrak{h})$ is

$$
G\left(F_{4, a}\right)=T(\mathfrak{h}) \oplus \bigoplus_{\xi \in G^{*}} \mathfrak{g}_{\xi},
$$

where $G^{*}=G \backslash 0$ and $G:=\langle\alpha, \beta, \gamma, \lambda\rangle$ is an elementary abelian group of order 16 and $\operatorname{dim}_{K}\left(\mathfrak{g}_{\xi}\right)=2$, for all $\xi \in G$.

Remark 6.3. The multiplication table of $G\left(F_{4, a}\right)$ on $K$ is given in Table 3 where the diagonal exhibits the elements $x^{[2]}$, for each $x \in \Phi$. For the sake of simplicity, we denote each class $\bar{x}=x+\tilde{G}\left(F_{4, a}\right)$ by its canonical representative $x$. For instance, we write $e_{1}$ instead $\overline{e_{1}}$. Other notations are the following: $h_{0}:=a h_{1}+h_{2}+h_{3}+h_{4}$, $h_{12}:=a h_{1}+h_{2}$, and $h_{\overline{4}}:=a h_{1}+h_{2}+h_{3}$. With $p_{\bar{i}}$ (resp., $q_{\bar{i}}$ ), we denote $p_{i_{1} i_{2} i_{3}}$ (resp., $q_{i_{1} i_{2} i_{3}}$ ), where $1 \leq i_{1}<i_{2}<i_{3} \leq 4$ and $i_{1}, i_{2}$, and $i_{3}$ are different from $i$. For instance, $p_{\overline{2}}$ is used instead of $p_{134}$.

\section{ACKNOWLEDGMENT}

The authors wish to thank this journal's editorial staff for their valuable help in improving the article. 


\begin{tabular}{|c|c|c|c|c|c|c|c|c|c|c|c|c|c|c|c|c|c|c|c|c|c|c|c|c|c|c|c|c|c|c|}
\hline & $e_{1}$ & $e_{2}$ & $e_{3}$ & $e_{4}$ & $f_{1}$ & $f_{2}$ & $f_{3}$ & $f_{4}$ & $p_{12}$ & $p_{13}$ & $p_{14}$ & $p_{23}$ & $p_{24}$ & $p_{34}$ & $q_{12}$ & \begin{tabular}{l|l}
$q_{13}$ \\
\end{tabular} & $q_{14}$ & $q_{23}$ & $q_{24}$ & $q_{34}$ & $p_{\overline{4}}$ & $p_{\overline{3}}$ & $p_{\overline{2}}$ & $p_{\overline{1}}$ & $p_{0}$ & $q_{\overline{4}}$ & $q_{\overline{3}}$ & $q_{\overline{2}}$ & $q_{\overline{1}}$ & $q_{0}$ \\
\hline$e_{1}$ & 0 & $p_{12}$ & $p_{13}$ & $p_{14}$ & $h_{1}$ & 0 & 0 & 0 & 0 & 0 & 0 & $p_{\overline{4}}$ & $p_{\overline{3}}$ & $p_{\overline{2}}$ & $f_{2}$ & 0 & 0 & 0 & 0 & 0 & 0 & 0 & 0 & $p_{0}$ & 0 & $q_{23}$ & $q_{24}$ & 0 & 0 & $q_{\overline{1}}$ \\
\hline$e_{2}$ & $p_{12}$ & 0 & $p_{23}$ & $p_{24}$ & 0 & $h_{2}$ & 0 & 0 & 0 & 0 & 0 & 0 & 0 & $p_{\overline{1}}$ & $a f_{1}$ & 0 & 0 & $f_{3}$ & 0 & 0 & $p_{13}$ & 0 & 0 & 0 & $p_{\overline{2}}$ & $a q_{13}$ & $a q_{14}$ & 0 & $q_{34}$ & $a q_{\overline{2}}$ \\
\hline$e_{3}$ & $p_{13}$ & $p_{23}$ & 0 & $p_{34}$ & 0 & 0 & $h_{3}$ & 0 & $p_{\overline{4}}$ & 0 & 0 & 0 & 0 & 0 & 0 & 0 & 0 & $f_{2}$ & 0 & $f_{4}$ & 0 & 0 & 0 & $p_{24}$ & $p_{\overline{3}}$ & $q_{12}$ & 0 & 0 & $q_{24}$ & $q_{\overline{3}}$ \\
\hline$e_{4}$ & $p_{14}$ & $p_{24}$ & $p_{34}$ & 0 & 0 & 0 & 0 & $h_{4}$ & $p_{\overline{3}}$ & $p_{\overline{2}}$ & 0 & $p_{\overline{1}}$ & 0 & 0 & 0 & 0 & 0 & 0 & 0 & $f_{3}$ & $p_{0}$ & 0 & 0 & 0 & 0 & 0 & 0 & $q_{13}$ & $q_{23}$ & $q_{\overline{4}}$ \\
\hline$f_{1}$ & $h_{1}$ & 0 & 0 & 0 & 0 & $q_{12}$ & $q_{13}$ & $q_{14}$ & $e_{2}$ & 0 & 0 & 0 & 0 & 0 & 0 & 0 & 0 & $q_{\overline{4}}$ & $q_{\overline{3}}$ & $q_{\overline{2}}$ & $p_{23}$ & $p_{24}$ & 0 & 0 & $p_{\overline{1}}$ & 0 & 0 & 0 & $q_{0}$ & 0 \\
\hline$f_{2}$ & 0 & $h_{2}$ & 0 & 0 & $q_{12}$ & 0 & $q_{223}$ & $q_{24}$ & $a e_{1}$ & 0 & 0 & $e_{3}$ & 0 & 0 & 0 & 0 & 0 & 0 & 0 & $q_{\overline{1}}$ & $a p_{13}$ & $a p_{14}$ & 0 & $p_{34}$ & $a p_{\overline{2}}$ & 0 & 0 & 0 & 0 & $q_{\overline{2}}$ \\
\hline$f_{3}$ & 0 & 0 & $h_{3}$ & 0 & $q_{13}$ & $q_{23}$ & 0 & $q_{34}$ & 0 & 0 & 0 & $e_{2}$ & 0 & $e_{4}$ & $q_{\overline{4}}$ & 0 & 0 & 0 & 0 & 0 & $p_{12}$ & 0 & 0 & $p_{24}$ & $p_{\overline{3}}$ & 0 & 0 & 0 & $q_{24}$ & $q_{\overline{3}}$ \\
\hline$f_{4}$ & 0 & 0 & 0 & $h_{4}$ & $q_{14}$ & $q_{24}$ & $q_{34}$ & 0 & 0 & 0 & 0 & 0 & 0 & $e_{3}$ & $q_{\overline{3}}$ & $q_{\overline{2}}$ & 0 & $q_{\overline{1}}$ & 0 & 0 & 0 & 0 & $p_{13}$ & $p_{23}$ & $p_{\overline{4}}$ & $q_{0}$ & 0 & 0 & 0 & 0 \\
\hline$p_{12}$ & 0 & 0 & $p_{\overline{4}}$ & $p_{\overline{3}}$ & $e_{2}$ & $a e_{1}$ & 0 & 0 & 0 & 0 & 0 & $p_{13}$ & 0 & $p_{0}$ & $h_{12}$ & 0 & 0 & 0 & 0 & 0 & 0 & 0 & 0 & $p_{\overline{2}}$ & 0 & $f_{3}$ & 0 & 0 & 0 & 0 \\
\hline$p_{13}$ & 0 & 0 & 0 & $p_{\overline{2}}$ & 0 & 0 & 0 & 0 & 0 & 0 & 0 & 0 & 0 & 0 & 0 & 0 & 0 & 0 & 0 & 0 & 0 & 0 & 0 & 0 & 0 & 0 & 0 & 0 & 0 & 0 \\
\hline$p_{14}$ & 0 & 0 & 0 & 0 & 0 & 0 & 0 & 0 & 0 & 0 & 0 & 0 & 0 & 0 & 0 & 0 & 0 & 0 & 0 & 0 & 0 & 0 & 0 & 0 & 0 & 0 & 0 & 0 & 0 & 0 \\
\hline$p_{23}$ & $p_{\overline{4}}$ & 0 & 0 & $p_{\overline{1}}$ & 0 & $e_{3}$ & $e_{2}$ & 0 & $p_{13}$ & 0 & 0 & 0 & 0 & $p_{24}$ & 0 & 0 & 0 & $h_{23}$ & 0 & 0 & 0 & 0 & 0 & 0 & 0 & $a f_{1}$ & 0 & 0 & $f_{4}$ & $a q_{14}$ \\
\hline$p_{24}$ & $p_{\overline{3}}$ & 0 & 0 & 0 & 0 & 0 & 0 & 0 & 0 & 0 & 0 & 0 & 0 & 0 & 0 & $p_{\overline{3}}$ & 0 & 0 & 0 & 0 & 0 & 0 & 0 & 0 & 0 & 0 & 0 & 0 & 0 & 0 \\
\hline$p_{34}$ & $p_{\overline{2}}$ & $p_{\overline{1}}$ & 0 & 0 & 0 & 0 & $e_{4}$ & $e_{3}$ & $p_{0}$ & 0 & 0 & $p_{24}$ & 0 & 0 & 0 & 0 & 0 & 0 & 0 & $h_{34}$ & $p_{\overline{3}}$ & 0 & 0 & 0 & 0 & 0 & 0 & 0 & 0 & $q_{12}$ \\
\hline$q_{12}$ & $f_{2}$ & $a f_{1}$ & 0 & 0 & 0 & 0 & $q_{\overline{4}}$ & $q_{\overline{3}}$ & $h_{12}$ & 0 & 0 & 0 & 0 & 0 & 0 & 0 & 0 & $q_{13}$ & 0 & $q_{0}$ & $e_{3}$ & 0 & 0 & 0 & 0 & 0 & 0 & 0 & $q_{\overline{2}}$ & 0 \\
\hline$q_{13}$ & 0 & 0 & 0 & 0 & 0 & 0 & 0 & $q_{\overline{2}}$ & 0 & 0 & 0 & 0 & 0 & 0 & 0 & 0 & 0 & 0 & 0 & 0 & 0 & 0 & 0 & 0 & 0 & 0 & 0 & 0 & 0 & 0 \\
\hline$q_{14}$ & 0 & 0 & 0 & 0 & 0 & 0 & 0 & 0 & 0 & 0 & 0 & 0 & 0 & 0 & 0 & 0 & 0 & 0 & 0 & 0 & 0 & 0 & 0 & 0 & 0 & 0 & 0 & 0 & 0 & 0 \\
\hline$q_{23}$ & 0 & $f_{3}$ & $f_{2}$ & 0 & $q_{\overline{4}}$ & 0 & 0 & $q_{\overline{1}}$ & 0 & 0 & 0 & $h_{23}$ & 0 & 0 & $q_{13}$ & 0 & 0 & 0 & 0 & $q_{24}$ & $a e_{1}$ & 0 & 0 & $e_{4}$ & $a p_{14}$ & 0 & 0 & 0 & 0 & 0 \\
\hline$q_{24}$ & 0 & 0 & 0 & 0 & $q_{\overline{3}}$ & 0 & 0 & 0 & 0 & 0 & 0 & 0 & 0 & 0 & 0 & 0 & 0 & 0 & 0 & $q_{24}$ & $a e_{1}$ & 0 & 0 & $e_{4}$ & $a p_{14}$ & 0 & 0 & 0 & 0 & $\begin{array}{ll} \\
\end{array}$ \\
\hline$q_{34}$ & 0 & 0 & $f_{4}$ & $f_{3}$ & $q_{\overline{2}}$ & $q_{\overline{1}}$ & 0 & 0 & 0 & 0 & 0 & 0 & 0 & $h_{34}$ & $q_{0}$ & 0 & 0 & $q_{24}$ & 0 & 0 & 0 & 0 & 0 & 0 & $p_{12}$ & $q_{\overline{3}}$ & 0 & 0 & 0 & 0 \\
\hline$p_{\overline{4}}$ & 0 & $p_{13}$ & 0 & $p_{0}$ & $p_{23}$ & $a p_{13}$ & $p_{12}$ & 0 & 0 & 0 & 0 & 0 & 0 & $p_{\overline{3}}$ & $e_{3}$ & 0 & 0 & $a e_{1}$ & 0 & 0 & 0 & 0 & 0 & 0 & 0 & $h_{\overline{4}}$ & 0 & 0 & 0 & $f_{4}$ \\
\hline$p_{\overline{3}}$ & 0 & 0 & 0 & 0 & $p_{24}$ & $a p_{14}$ & 0 & 0 & 0 & 0 & 0 & 0 & 0 & 0 & 0 & 0 & 0 & 0 & 0 & 0 & 0 & 0 & 0 & 0 & 0 & 0 & 0 & 0 & 0 & 0 \\
\hline$p_{\overline{2}}$ & 0 & 0 & 0 & 0 & 0 & 0 & 0 & $p_{13}$ & 0 & 0 & 0 & 0 & 0 & 0 & 0 & 0 & 0 & 0 & 0 & 0 & 0 & 0 & 0 & 0 & 0 & 0 & 0 & 0 & 0 & 0 \\
\hline$p_{\overline{1}}$ & $p_{0}$ & 0 & $p_{24}$ & 0 & 0 & $p_{34}$ & $p_{24}$ & $p_{23}$ & $\overline{p_{\overline{2}}}$ & 0 & 0 & 0 & 0 & 0 & 0 & 0 & 0 & $e_{4}$ & 0 & 0 & 0 & 0 & 0 & 0 & 0 & 0 & 0 & 0 & $h_{\overline{1}}$ & $a f_{1}$ \\
\hline$p_{0}$ & 0 & $p_{\overline{2}}$ & $p_{\overline{3}}$ & 0 & $p_{\overline{1}}$ & $a p_{\overline{2}}$ & $p_{\overline{1}}$ & $p_{\overline{4}}$ & 0 & 0 & 0 & 0 & 0 & 0 & 0 & 0 & 0 & $a p_{14}$ & 0 & $p_{12}$ & 0 & 0 & 0 & 0 & 0 & $e_{4}$ & 0 & 0 & $a e_{1}$ & $h_{0}$ \\
\hline$q_{\overline{4}}$ & $q_{23}$ & $a q_{13}$ & $q_{12}$ & 0 & 0 & $q_{13}$ & 0 & $q_{0}$ & $f_{3}$ & 0 & 0 & $a f_{1}$ & 0 & 0 & 0 & 0 & 0 & 0 & 0 & $q_{\overline{3}}$ & $h_{\overline{4}}$ & 0 & 0 & 0 & $e_{4}$ & 0 & 0 & 0 & 0 & 0 \\
\hline$q_{\overline{3}}$ & $q_{24}$ & $a q_{14}$ & 0 & 0 & 0 & 0 & 0 & 0 & 0 & 0 & 0 & 0 & 0 & 0 & 0 & 0 & 0 & 0 & 0 & 0 & 0 & 0 & 0 & 0 & 0 & 0 & 0 & 0 & 0 & 0 \\
\hline$q_{\overline{2}}$ & 0 & 0 & 0 & $q_{13}$ & 0 & 0 & 0 & 0 & 0 & 0 & 0 & 0 & 0 & 0 & 0 & 0 & 0 & 0 & 0 & 0 & 0 & 0 & 0 & 0 & 0 & 0 & 0 & 0 & 0 & 0 \\
\hline$q_{\overline{1}}$ & 0 & $q_{34}$ & $q_{24}$ & $q_{23}$ & $q_{0}$ & 0 & $a q_{24}$ & 0 & 0 & 0 & $f_{4}$ & 0 & 0 & 0 & $q_{\overline{2}}$ & 0 & 0 & 0 & 0 & 0 & 0 & 0 & 0 & $h_{\overline{1}}$ & $a e_{1}$ & 0 & 0 & 0 & 0 & 0 \\
\hline$q_{0}$ & $q_{\overline{1}}$ & $a q_{\overline{2}}$ & $q_{\overline{3}} \bar{z}$ & $q_{\overline{4}}$ & 0 & $q_{\overline{\overline{2}}}$ & $q_{\bar{z}}$ & 0 & 0 & 0 & 0 & $a q_{1}$ & 0 & $q_{12}$ & & 0 & 0 & 0 & 0 & 0 & $f_{4}$ & 0 & & $a f_{1}$ & $h_{0}$ & & & & 0 & \\
\hline
\end{tabular}

TABLE 3. Multiplication table of $G\left(F_{4, a}\right)$. 


\section{REFERENCES}

[1] S. P. Demuškin, Cartan subalgebras of the simple Lie p-algebras $W_{n}$ and $S_{n}$, Sibirsk. Mat. Ž. 11 (1970), 310-325. MR 0262310.

[2] A. Grishkov and A. Premet, Simple Lie algebras of absolute toral rank 2 in characteristic 2, Preprint. https://www.ime.usp.br/ grishkov/papers/asp.pdf

[3] A. Grishkov, On simple Lie algebras over a field of characteristic 2, J. Algebra 363 (2012), 14-18. MR 2925843.

[4] G. M. D. Hogeweij, Almost-classical Lie algebras. I, II, Nederl. Akad. Wetensch. Indag. Math. 44 (1982), no. 4, 441-452, 453-460. MR 0683531

[5] N. Jacobson, Lie algebras, Interscience Tracts in Pure and Applied Mathematics, No. 10, Interscience Publishers, New York, 1962. MR 0143793

[6] N. Jacobson, Abstract derivation and Lie algebras, Trans. Amer. Math. Soc. 42 (1937), no. 2, 206-224. MR 1501922

[7] V. G. Kac, The classification of the simple Lie algebras over a field with non-zero characteristic, Izv. Akad. Nauk SSSR Ser. Mat. 34 (1970), 385-408. MR 0276286

[8] I. Kaplansky, Linear algebra and geometry. A second course, Allyn and Bacon, Boston, MA, 1969. MR 0249444

[9] G. B. Seligman, On Lie algebras of prime characteristic, Mem. Amer. Math. Soc. 19 (1956). MR 0077876

[10] S. Skryabin, Toral rank one simple Lie algebras of low characteristics, J. Algebra 200 (1998), no. 2, 650-700. MR 1610680

[11] R. Steinberg, Automorphisms of classical Lie algebras, Pacific J. Math. 11 (1961), 1119-1129. MR 0143845

[12] H. Strade, The absolute toral rank of a Lie algebra, in Lie algebras, Madison 1987, 1-28, Lecture Notes in Math., 1373, Springer, Berlin, 1989. MR 1007321.

[13] B. Ju. Vě̆sfeřler and V. G. Kac, Exponentials in Lie algebras of characteristic p, Izv. Akad. Nauk SSSR Ser. Mat. 35 (1971), 762-788. MR 0306282.

Carlos R. Payares Guevara ${ }^{凶}$

Facultad de ciencias básicas, Universidad Tecnológica de Bolívar, Cartagena de Indias, Colombia cpayares@utb.edu.co

Fabián A. Arias Amaya

Faculty of basic sciences, Universidad Tecnológica de Bolívar, Cartagena de Indias, Colombia

farias@utb.edu.co

Received: March 20, 2019

Accepted: December 26, 2019 